\title{
Two Kind of Measles-Related Encephalitis in Pediatric Patients: A Case Report
}

ISSN: 2639-0531

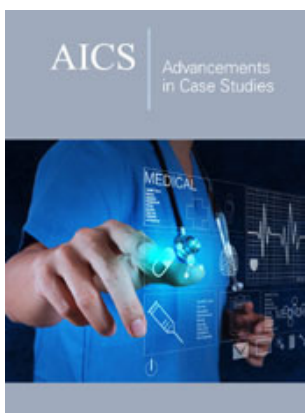

*Corresponding author: Yevtushenko V, 0 Bogomolets National Medical University, Ukraine

Submission: 棒October 10, 2019

Published: 㘹October 17, 2019

Volume 2 - Issue 2

How to cite this article: Yevtushenko V, Two Kind of Measles-Related Encephalitis in Pediatric Patients: A Case Report. Adv Case Stud.2(1). AICS.000532.2019.

Copyright@ Yevtushenko V, This article is distributed under the terms of the Creative Commons Attribution 4.0 International License, which permits unrestricted use and redistribution provided that the original author and source are credited.

\author{
Yevtushenko $\mathrm{V}^{*}$ and Kramarov S \\ O Bogomolets National Medical University, Ukraine
}

\begin{abstract}
Background: Measles remains one of the leading causes of death among children around the world. Encephalitis is the most serious complication of measles virus infection.

Case presentation: We report two measles-induced encephalitis who was admitted to to Kyiv City Pediatric Infectious Clinical Hospital. One patient had postinfectious variant of measles encephalitis that occurred 6 days after primary measles infection onset. Disease had benign course with substantial improvement during hospital stay. Another patient presented with subacute sclerosing panencephalitis. Neurological symptoms started 10 years after measles infection. Disease characterized by progredient course and unfavorable outcome.
\end{abstract}

Conclusion: Measles encephalitis is rare but serious and often deadliest complication. It should be considered in differential diagnostic of CNS infection among pediatric patients.

Keywords: Measles, Encephalitis, Children

\section{Introduction}

Encephalites have a wide range of etiological factors. The measles virus is relatively rare during large-scale vaccination. However, during the outbreak, its role increases, and it should be considered in the differential diagnosis of encephalitis. A major measles outbreak continues in Ukraine during 2017 and up to now, with over 100,000 patients and dozens dead. Encephalitis is the most serious complication of measles virus infection [1].

\section{Methods}

We retrospectively collected data from two patients with measles associated encephalitis who were admitted to Kyiv City Pediatric Infectious Clinical Hospital (KCPICH) throughout the year 2018.

\section{Results}

In 2018, we observed two patients with measles associated CNS infection. Among them, a girl of 5.5 years old, unvaccinated against measles, whose disease started suddenly with a temperature rise to febrile values, the appearance of catarrhal phenomena. At the 3rd day of the disease, a maculopapular rash appeared on the face that spread throughout the body during the next 24 hours. On the 5th day, the general condition of the child worsened, she became sleepy, refused to eat, could not rise from the bed without help, pain in the legs and back appeared, while the rash on the skin at that time disappeared. On the 6 th day of the disease examination revealed an impairment of consciousness (score 13 based on the Glasgow coma scale, E4V4M5); the physical examination showed positive meningeal symptoms (rigidity of the occipital muscles, Kernig's and Brudzinski's signs), symptoms of spinal nerve roots tension (Neri's sign). The child was not accessible for the productive contact. The face was symmetrical. The bulbar group of the cranial nerves was not impaired. Pupils $D=S$, moderately expanded, photoreaction was preserved, conjugate. General muscular hypotonia. Abdominal reflexes were absent. There was no seizures and vomit. Body temperature was $36.4-36.6{ }^{\circ} \mathrm{C}$. Microcirculation was not affected. Breathing was spontaneous, effective, biomechanics was not impaired, with the support of humidified oxygen through a face mask. Heart rate was 58$86 / \mathrm{min}$, blood pressure was 103/41-83/48 mmHg. Peristalsis was preserved. There was no self-defecation. Diuresis was at $3 \mathrm{ml} / \mathrm{kg} / \mathrm{hr}$. During the next 3 days, the state of consciousness deteriorated. On the day 9 of the disease, the level of consciousness was 9 based on the Glasgow 
coma scale (E4V1M4), bradycardia increased to $44 / \mathrm{min}$, there was a decrease in blood pressure to $65 / 40 \mathrm{mmHg}$. Cerebrospinal fluid analysis revealed slight pleocytosis (25 lymphocytic cells), while a MRI study showed scattered focal changes in white and gray matter. From the day 10 of the disease, there was a gradual improvement of the child's condition. Restoration of consciousness was observed on the day 12. On the day 8 of the disease, a result of the study of IgM the measles virus was obtained. During discharge from the hospital on the day 18 of the disease, the child had minor manifestations persisted in the form of a decrease in muscular strength in the limbs, increased fatigue.

Another child, an 11-year-old girl, was hospitalized to the KCCH with an impairment of consciousness (score 9 according to the Glasgow coma scale, E4V1M4). No impairments of the cranial nerves were observed. Abdominal reflexes were not activated. Muscle tone was lowered. Breathing was spontaneous, effective. Hemodynamics was stable. The disease history showed that the child suffered from measles at the age of 9 months old. 1.5 years ago, the girl suddenly began having her motor function impaired in the form of spontaneous tremor of hands, inclination of her head to the right, episodes of involuntary falls up to 30 times a day. Subsequently, there were periods of deterioration and improvement of her neurological status. Four days before hospitalization in our clinic, the child became sleepier, the intensity of myoclonus and hallucinatory reactions increased. On the day of hospitalization, the child had consciousness impaired to the level of a sopor (score 9 according to the Glasgow coma scale, E4V1M4), and lymphocytic pleocytosis (78 lymphocytes) was detected in the cerebrospinal fluid. MRI of the brain showed signs of periventricular leukoaraiosis. The results of the test of the cerebrospinal fluid for the markers of toxoplasmosis, CMV, EBV, VZV, HSV1/2, HHV 6-7 (PCR) were negative, but a high titer of IgG antibody to measles virus was detected. The child received empirical antiviral therapy (acyclovir), immunomodulatory therapy (corticosteroids, intravenous immunoglobulin), supportive and symptomatic agents. However, despite the treatment, the condition of the child progressively deteriorated, with the gradual development of multiple organ failure. The patient died 18 days after being admitted to the ICU.

\section{Discussion}

Measles-induced encephalitis may present as acute primary or postinfectious encephalitis, measles inclusion body encephalitis
(MIBE) and as a progressive neurological disorder (subacute sclerosing panencephalitis, SSPE) [2]. The first case had features of acute post-measles encephalitis. Signs of neurological complication began at the end of the first week. At that time the acute manifestations of the measles disease had already subsided. The case of measles was confirmed by positive IgM test and no other responsible pathogens was revealed. Cerebrospinal fluid changes and brain MRI findings was also typical for postinfectious encephalitis [3]. It was relatively benign course of encephalitis with fast improvement within three weeks. In opposite to the previous case second one was the more difficult challenge. Initial examination revealed no common causative agent, no direct connection with preceded infection, and signs of some immunodeficiency status was absent. Suspicion about SSPE was raised due to medical history consisted attacks of neurological disorders. This case fit two big Dyken's criteria for SSPE and white matter hyperintensities on MRI [4]. Disease began 10 years after episode of measles in early childhood. SSPE is usually a progressive disorder and death usually occurs in 1-3 years. Just $5 \%$ of the patients can have substantial spontaneous long term improvement [5]. Our case also had unfavorable outcome despite intensive treatment.

\section{Conclusion}

Measles encephalitis is rare but serious and often deadliest complication. It should be considered in differential diagnostic of CNS infection among pediatric patients.

\section{References}

1. Lancella L, Di Camillo C, Vittucci AC, Boccuzzi E, Bozzola E, et al. (2017) Measles lessons in an anti-vaccination era: public health is a social duty, not a political option. Ital J Pediatr 43(1): 102.

2. Al Qayoudhi A, Al Kindi H, Meki N, Al Maani A (2016) Acute measles encephalitis in an immigrant Syrian child: Case report and review of the literature. Oman Med J 31(2): 150-153.

3. Bale JF (2015) Virus and immune-mediated encephalitides: Epidemiology, diagnosis, treatment, and prevention. Pediatr Neurol 53(1): 3-12.

4. Jafri SK, Kumar R, Ibrahim SH (2018) Subacute sclerosing panencephalitis-current perspectives. Pediatr Heal Med Ther 9: 67-71.

5. Garg RK (2002) Subacute sclerosing panencephalitis. Postgrad Med J 78(916): 63-70

For possible submissions Click below:

Submit Article 